\title{
Research of Dispatching Method in Elevator Group Control System Based on Fuzzy Neural Network
}

\author{
Yufeng Dai ${ }^{\text {a }}$, Yun Du ${ }^{\text {b }}$ \\ School of electrical engineering, Hebei University of Science and Technology, Shijiazhuang 050018, \\ China \\ adai393158117@163.com, b379269095@qq.com
}

\begin{abstract}
Keywords: Elevator group control system, Comprehensive evaluation function, Fuzzy neural network, Dispatching method.
\end{abstract}

\begin{abstract}
Elevator group control system (EGCS) is a complex optimization system to with multiobjective, stochastic and nonlinear characteristics. It is hard to describe EGCS with exact mathematic model and to increase the capability of the system with traditional control method. The fuzzy control technology and neural network technology are combined in this paper and a dispatching method applied to various passenger traffic conditions is proposed. The comprehensive evaluation function of traffic signal is established and the right heavy of every evaluation factor (waiting time, riding time, energy consume) is studied by the neural network, so the elevator is dispatched optimally. The result of simulation shows that this method realizes reasonable elevator dispatching under various passenger traffic conditions and indicates the validity of this method.
\end{abstract}

\section{Introduction}

With the continuous development and progress of human society, the number of high-rise buildings has become a symbol of a city's development and prosperity, and the elevator is an indispensable part of the high-rise building, so the elevator technology rapidly developed. Elevator in the control technology gradually developed from a lift to the centralized control of elevators. First of all, through the analysis of building passenger flow, the traffic pattern of elevator group is classified. Fuzzy neural network is used to identify the traffic pattern of elevator group. According to the identification of the system to determine the elevator group is currently in the traffic model. Then, the fuzzy neural network is used to calculate the credibility of the elevator in response to the call signal of the elevator, and the elevator with the highest reliability is selected to finally complete the service [6].

\section{Classification and identification of traffic patterns}

\subsection{The classification of the traffic patterns.}

According to the different needs of the building and the people who work in the building are also different, so there are a variety of traffic patterns corresponding to them [2].In this paper, the high-rise office building as the research object, ac-cording to the fixed time traffic flow changes, the elevator traffic model is divided into 6 categories: up peak traffic pattern, down peak traffic pattern, idle traffic mode, the middle layer of busy mode, single mode and double layer busy mode.

\subsection{Fuzzy neural network.}

After the classification of traffic patterns, we need to identify the current traffic pattern of elevator group. In this paper, fuzzy neural network is used to identify. In the fuzzy neural network structure, the number of layers is fixed, which are input layer, fuzzy layer, rule layer, integrated layer and output layer [5].

(1) Input layer: the input layer is the first layer of the fuzzy neural network topology, each node in the input layer represents an input variable, and the number of neurons is equal to the number of input variables. 
(2) Fuzzy level: each input variable should be defined by their fuzzy subsets, and the membership functions of all fuzzy subsets are calculated by this layer.

(3) Rule layer: the node is a rule node, which represents the logic rules, and each node has the logic operation function.

(4) Synthesis layer: this layer node performs a fuzzy "or" operation to synthesize rules with the same result.

(5) Output layer: This layer is also called the anti-fuzzy layer.

\subsection{Traffic pattern recognition.}

The high-rise office building as an example, in 5 minutes as a unit of time, characteristic values are: unit time total passenger traffic, passenger flow, passenger flow into the left, the largest passenger flow, the middle floor large middle floor. After normalization, the input variables belong to $[0,1]$. The fuzzy neural network needs to be trained before pattern recognition, and the recognition can be carried out after the training [1]. Because the training input data need to be normalized, the input value can only be in the $[0,1]$, so the input sample interval value is 0.2 . The structure of fuzzy neural network in traffic pattern recognition is 3-18-216-24-4 and 2-8-16-12-3.

According to the previous learning methods to train the network, the fuzzy rule extraction threshold is $=0.05$. Network training results is as Table 2.1.

Table 1 Network training results

\begin{tabular}{|c|c|c|c|c|}
\hline Network type & $\begin{array}{c}\text { Learning } \\
\text { efficiency }\end{array}$ & Rule base & $\begin{array}{c}\text { Error back } \\
\text { propagation }\end{array}$ & Error precision \\
\hline First step & 0.01 & 126 & 123 & 0.000997 \\
\hline Second step & 0.01 & 12 & 37 & 0.000995 \\
\hline
\end{tabular}

It can be seen from the table that the rules are deleted and merged by competitive learning. Now, the trained fuzzy neural network is used to identify the traffic pattern of the office building. The simulation results are as Fig 1-4.

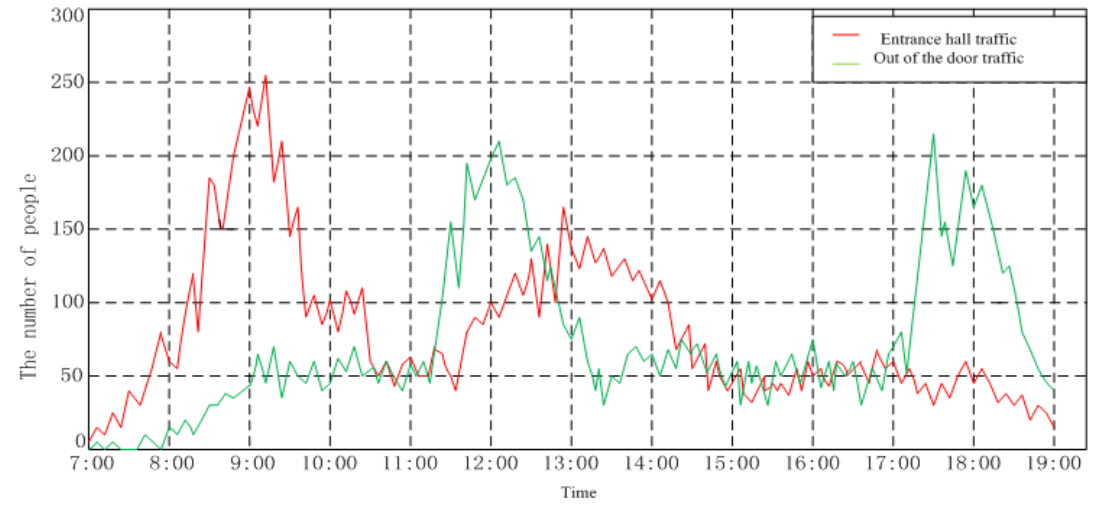

Fig.1 Traffic statistics of the hall

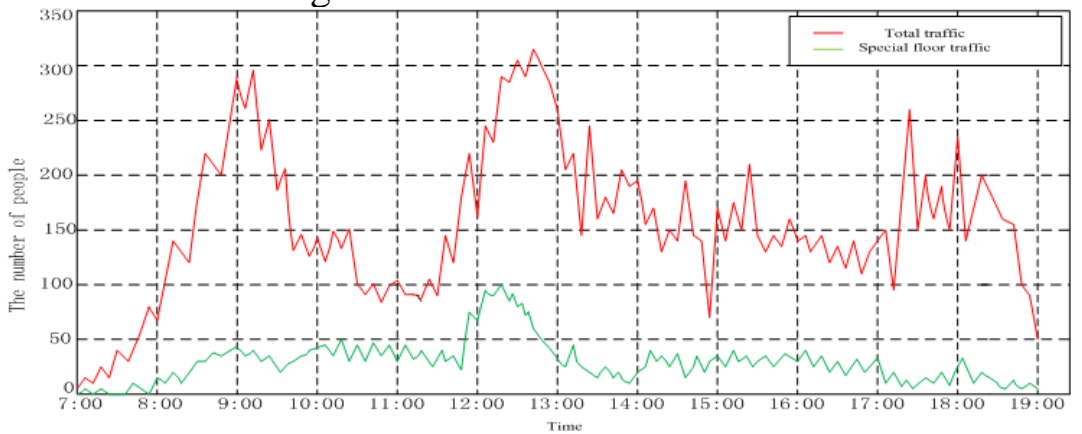

Fig. 2 The total traffic volume of the office building and its special floor traffic characteristics 


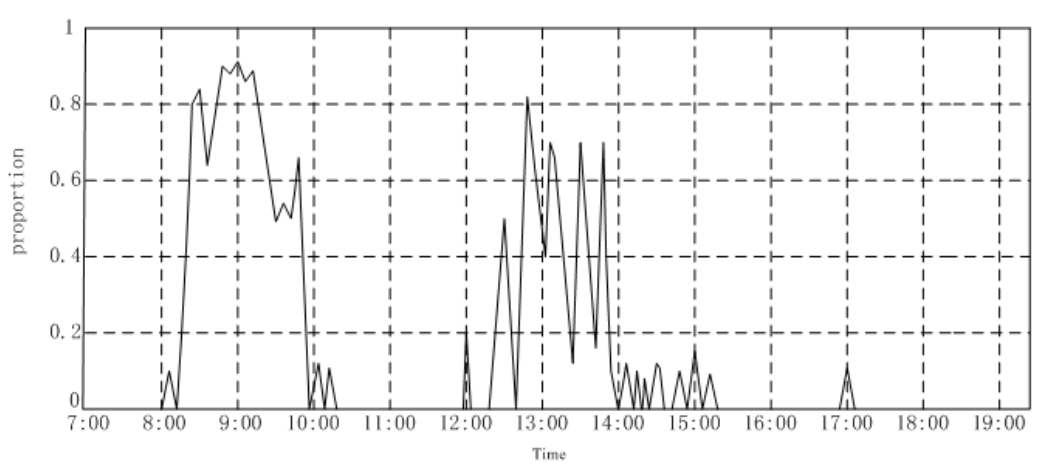

Fig. 3 The proportion of the up peak pattern

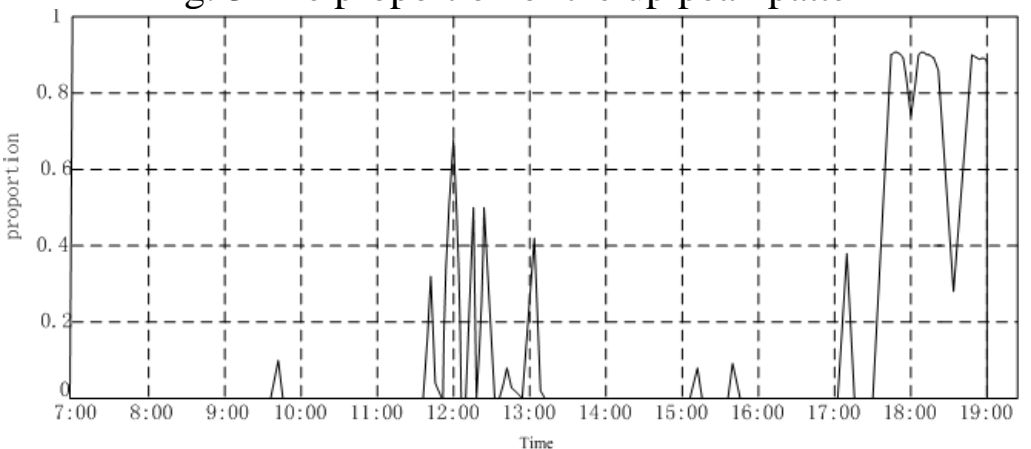

Fig. 4 The proportion of the downward peak mode

Figure 1 and Figure 2 is a traffic statistical feature curve of a working day in an office building, and the statistical time interval is 5 minutes. Using fuzzy neural network to identify traffic in Fig 1 and Fig 2, it identifies the proportion of up peak pattern and down peak pattern as shown in Fig 3 and Fig 4.

\section{Elevator group control system ladder algorithm}

The elevator group dispatch algorithm is a multi-objective optimization. It needs to consider the entire population and the average waiting time, average operation time of all people in the elevator, the time of waiting and the proportion of the overall energy consumption of elevator group and other factors. The elevator group control system needs to control multiple elevators to respond to the various calling signals at the same time, so the general mathematical model cannot be achieved [4]. We will continue to use fuzzy neural network to achieve multi-objective optimization.

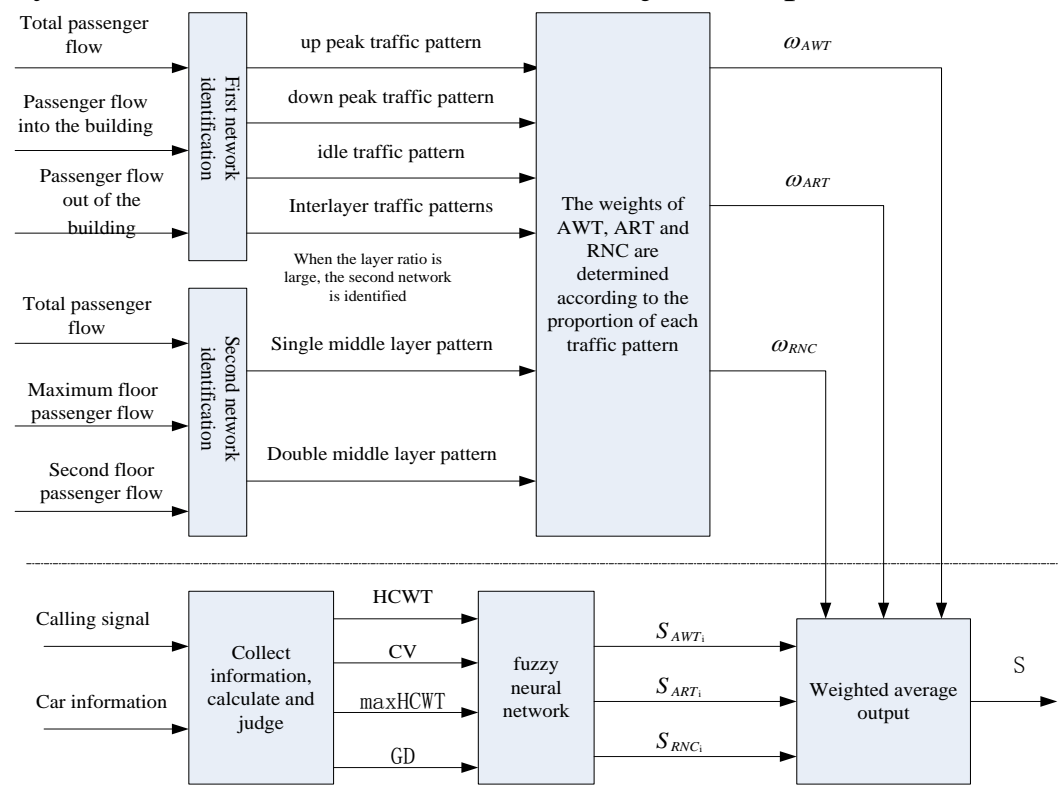

Fig.5 Schematic diagram of elevator group control 
This elevator performance of multiple indicators, the most importance is the passengers' average waiting time AWT, the average passenger elevator time ART and energy consumption RNC these three indicators. Objective function:

$$
S_{\mathrm{i}}=\omega_{A W T} S_{A W T_{\mathrm{i}}}+\omega_{A R T} S_{A R T_{\mathrm{i}}}+\omega_{R N C} S_{R N C_{\mathrm{i}}}
$$

Where $S_{\text {AWTi }}$ is the confidence of the average waiting time of the elevator, $S_{\text {ARTi }}$ is the confidence of the average ride time, and $S_{\text {Rर }}$ is the confidence of the energy loss. Indicates the reliability of the i-th elevator response call. $\omega_{A W T} 、 \omega_{A R T} 、 \omega_{R N C}$ is the weighting factor, and the sum is 1 .The weighting factor is determined by the pattern of traffic flow identified in the previous chapter. With the formula (1), the reliability of the $n$ elevators in response to the call signal can be obtained, and then the elevator with the highest degree of confidence is selected to respond to the call signal.

$$
\boldsymbol{S}_{\mathrm{e}}=\max \left(\boldsymbol{S}_{1}, \boldsymbol{S}_{2}, \ldots, \boldsymbol{S}_{\mathrm{n}}\right)
$$

When a call signal generated, the system will immediately through the HCWT, maxHCWT, CV and GD these four parameters to calculate the average waiting time for each elevator elevator AWT, the average passenger elevator time ART and energy consumption RNC indicators [3]. The HCWT generates a call signal to respond to the elevator's arrival time and waiting time for the layer. maxHCWT is the maximum waiting time for all call signals that a elevator responds to.CV rep-resents the ability to respond to future calls. GD represents the shortest distance between the newly generated call signal floor and all signal floors that the elevator responds to [7].

The intra-vector components are the weights of AWT, ART, and RNC, respectively. The traffic ratio of the up peak traffic pattern, down peak traffic pattern, interlayer traffic pattern, idle traffic pattern, single middle layer pattern and double middle layer pattern are $A_{1}, A_{2}, A_{3}, A_{4}, A_{5}, A_{6}$ respectively. Also there $A_{1}+A_{2}+A_{3}+A_{4}+A_{5}+A_{6}=1$. Then the final calculation of the AWT, ART, RNC weights are as follows:

$$
\varpi=A_{1} \omega_{1}+A_{2} \omega_{2}+A_{3} \omega_{3}+A_{4} \omega_{4}+A_{5} \omega_{5}+A_{6} \omega_{6}
$$

The fuzzy neural network model is established. The input variables are HCWT, max HCWT, CV and GD, and the output variables are AWT, ART and RNC. According to different modes of transport, it can adjust the weight of the three confidence, calculate the final credibility. By comparing the reliability of the elevator in response to the call signal, select the most reliable elevator to complete the escalator.

\section{Elevator group control system simulation}

In this paper, according to the actual situation of a certain office building statistics training data, is got considering the time period and the mode of traffic flow. The sample data were collected in 50 groups. The data of the 40 groups were training data, and the remaining 10 groups of data were test data. The error precision is controlled within the preset threshold by repetitive learning training, e $<0.001$. Figure 6 shows that in the training samples, the center and width of the membership function of the input vector of the fuzzy neural network by the error back propagation algorithm is optimized. If you follow the "checklist" your paper will conform to the requirements of the publisher and facilitate a problem-free publication process.

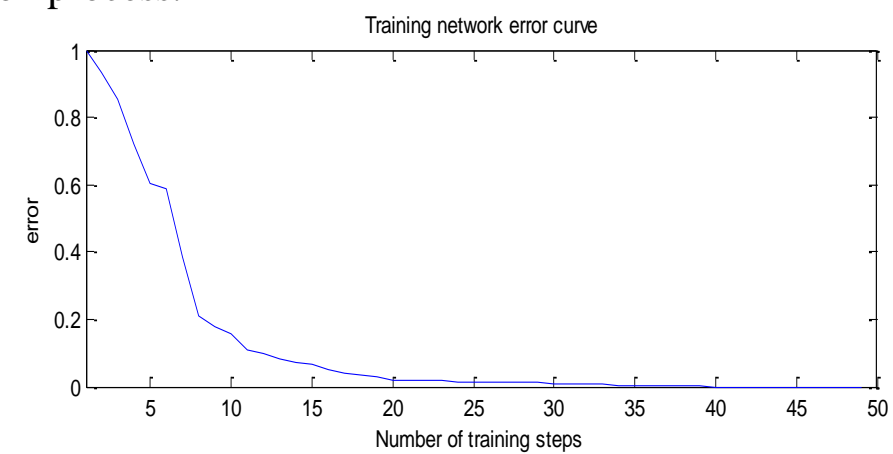

Fig. 6 Variation of network error performance during training 
It can be seen that after 30 iterations, the gradient curve has stabilized, indicating that the training of the fuzzy neural network has been completed. After the end of the network training, use the test sample to test the output error range, as shown in Figure 7.

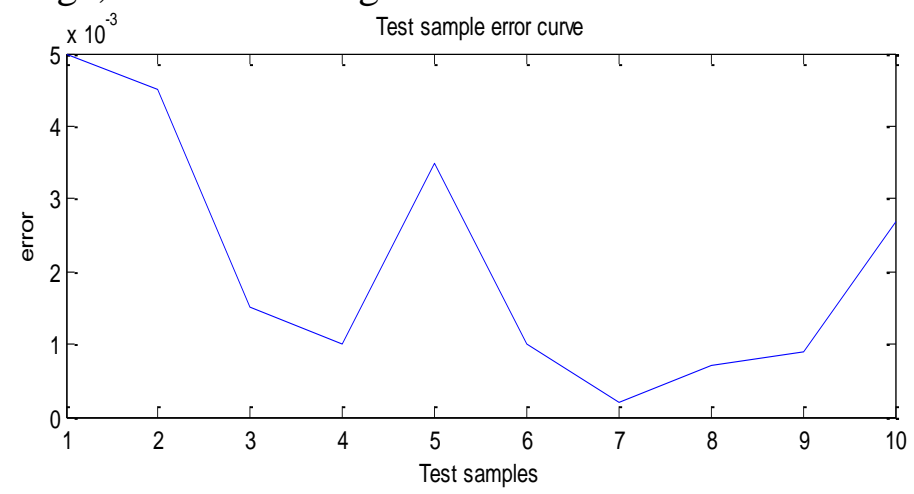

Fig. 7 Error result of test sample data

It can be seen from Figure 7 that the errors are within the set range, indicating that the fuzzy neural network training is completed.

\section{Conclusion}

In this paper, a 20-story building elevator group as an example of the simulation, the building has six elevators, each elevator rated 15 people. AWT, ART and RNC weights are calculated according to the current traffic pattern. The parameters of AWT, ART and RNC are calculated by HCWT, max HCWT, CV and GD. AWT, ART and RNC are calculated according to the current traffic pattern. , RNC three of the credibility, according to the traffic model to calculate the final credibility. By comparing the size of the final reliability of the six elevators, select the most reliable elevator to send ladder. In this paper, the simulation experiment is carried out by using MATLAB software, and the experimental results are obtained. How to adjust the weighting system reasonably and make the algorithm more optimized.

\section{References}

[1]. Bao H: Research on Fuzzy Neural Network Based recognition of traffic patterns of Elevator Group Control System and multi-target dispatching algorithm.[D] Tongji University, China 2007.

[2]. Guo JL: Research on Elevator Group Control System Based on Fuzzy Neural Network. [D]Northeastern University, China 2013.

[3]. Lu XY,He P,Chang JH : Research of Dispatching Method in Elevator Group Control System Based on Fuzzy Neural Network. Computer Technology and Development. (2008)18(1):220-222

[4]. Li XL: Studies of Elevator Group Control System Based on Fuzzy Neural Network. [D] Soochow University, China 2006.

[5]. Tang HY: Research on optimal Control of Elevator Group Based on Fuzzy Neural Network . Harbin Institute of Technology, [D] China 2006.

[6]. Wang QX,Jin X :Research on Elevator Group Control Algorithm based on Fuzzy Neural Network. China New Technologies and Products2015,9(2):4-5

[7]. Zhang SQ Application Research of Fuzzy Neural Network in Elevator Group Control system. Northeastern University, [D] China2009. 